\title{
Clinical case discussions - Hypopituitarism and pituitary apoplexy caused by pituitary Adenomas
}

\author{
Gaisheng Tian *, Qinxue Li, Dan Zhao, Ying Wang, Ruijing Yan \\ Department of Geriatrics, the Third Affiliated Hospital of Inner Mongolia Medical University, Baotou, China
}

Received: October 12, 2012

DOI: $10.14725 /$ dcc.v2n1p14
Accepted: October 28, $2012 \quad$ Online Published: March 15, 2015

URL: http://dx.doi.org/10.14725/dcc.v2n1p14

\begin{abstract}
A case of "Hypopituitarism and pituitary apoplex induced by pituitary Adenomas" was collected in the Third Affiliated Hospital of Inner Mongolia medical University Geriatric Department. A rare and atypical disease as it is, delayed diagnosis and treatment is easily seen among those cases. Therefore, the paper aims to investigate the difference of clinical characteristics and treatment outcome of pituitary adenomas in elder patient through the case analysis. Clinically, patients with pituitary dysfunction may have life-threatening crisis so that timely diagnosis and aggressive treatment can be effective in reducing the pituitary crisis and reducing the misdiagnosis rate.
\end{abstract}

Key Words: Pituitary tumor, Pituitary dysfunction, Pituitary apoplexy

\section{Medical record}

\subsection{General information}

A 75-year-old man who complained of intermittent weakness, dizziness, nausea, vomiting of 2 month's duration was admitted to our hospital on November 13, 2010. He had intermittent weakness, dizziness, transient amaurosis during body position changes 2 months prior his admission. Blood pressure was repeatedly measured to be low, 80-90/50-55 mmHg. He felt morning nausea, vomiting, loss of appetite. However, no headache, rash, epistaxis and gum bleeding, no cough and chest tightness, shortness of breath, abdominal pain, diarrhea, no vomiting, bloody diarrhea, joint pain or activity limitation, urine abnormal was manifested. The laboratory test results at the outpatient service included: blood $\mathrm{K}^{+} 4.3 \mathrm{mmol} / \mathrm{L}, \mathrm{Na}^{+} 127 \mathrm{mmol} / \mathrm{L}$, $\mathrm{Cl}^{-} 90 \mathrm{mmol} / \mathrm{L}$. Specific past medical history and family medical history were denied.

\subsection{Physical examination}

Data on the physical examination revealed his temperature $36.8^{\circ} \mathrm{C}$, Blood pressure $85 / 55 \mathrm{mmHg}$. He was poor spirited with pale appearance, smooth breathing, and dry skin, systemic superficial lymph node was not swollen and no rash was discovered. He had sparse pubic and armpit hair. Palm and gum pigmentation were not obvious. Left lung breath sounds resonance, while the other side sound decreased. The heart rate was 63 times per minute, showing regularity in the heartbeat. His abdomen was soft. No enlargement of liver, spleen and kidneys beneath the rib was found. No edema was seen in lower extremity, or redness, swelling, heat, pain, limited mobility was accompanied. Besides that, nervous system was normal.

\subsection{Auxiliary examination}

Pituitary adenoma was indicated by pituitary MRI (see Figure 1). Adrenal CT was normal; $\mathrm{Na}^{+} 124-131 \mathrm{mmol} / \mathrm{L}, \mathrm{Cl}^{-}$

\footnotetext{
*Correspondence: Gaisheng Tian; E-mail: nmgbgyyyy@163.com; Address: Department of Geriatrics, the Third Affiliated Hospital of Inner Mongolia Medical University, Baotou, China.
} 
98.0-90.9 mmol/L, K $+4.2-4.6 \mathrm{mmol} / \mathrm{L}, \mathrm{ACTH}<5.00 \mathrm{ng} / \mathrm{L}$, low cortisol rhythm, $2.1 \mu \mathrm{g} / \mathrm{dl}$ at $6-8$ o'clock, $0.6 \mu \mathrm{g} / \mathrm{dl}$ at 16-18 o'clock, $4.3 \mu \mathrm{g} / \mathrm{dl}$ at $23-01$ o'clock. Thyroid function test results: T3, TSH normal, T4 $59.2 \mathrm{nmol} / \mathrm{L}$, belownormal. The diagnosis of pituitary adenoma secondary hypothyroidism was then established. Prolactin, FSH, growth hormone were all normal. Fundus examination result: the double-field temporal defect, intraocular pressure was normal; urine specific gravity: 1.015-1.025.

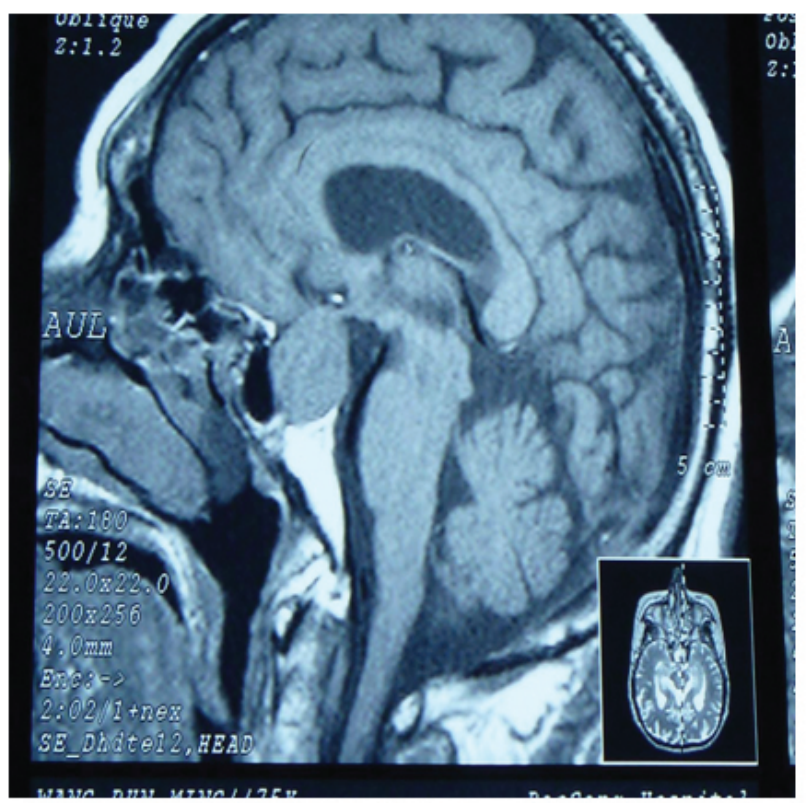

Figure 1: Pituitary MRI

\subsection{Primary diagnosis}

(1) Pituitary adenoma

(2) Anterior pituitary hypofunction

(3) Secondary adrenal hypofunction

(4) Hypothyroidism

\subsection{Diagnosis and treatment}

Blood pressure was measured to be $80-90 / 50-55 \mathrm{mmHg}$ after admission. The patient and his family refused to take surgery or radiation therapy. He received replacement therapy of hydrocortisone $20 \mathrm{mg} /$ day in the morning, plus euthyrox $25 \mu \mathrm{g}$ /day two weeks later. His condition became better after the treatment as fatigue, dizziness, nausea, vomiting disappeared, appetite and mental improved significantly. Blood pressure was measured to rise, 90-95/55-65 mmHg and $\mathrm{Na}^{+} 133-140 \mathrm{mmol} / \mathrm{L}, \mathrm{Cl}^{-}$95.0$99 \mathrm{mmol} / \mathrm{L}$. He was discharged when the reexamination result of thyroid function was normal. Long-term oral administration of hormones was required and increased amount of hydrocortisone in case of colds and other stress was needed.
He was readmitted to our hospital due to sudden blindness of the left eye and confusion of two hours' duration on December 19, 2010, three days after the deactivation of hydrocortisone. Physical examination results included: body temperature $36.7^{\circ} \mathrm{C}$, blood pressure $80 / 50 \mathrm{mmHg}$, heart rate of 120 beats/min, breathing 18 times/min, drowsiness, lung breath sounded rough, heart rate 68 beats/min, showing regularity in the force and rhythm of the heartbeat, abdomen was soft. Pathology was not evident. Reexamination results of pituitary MRI highly hinted of pituitary adenoma, pituitary apoplexy (PA) (see Figure 2) so that glucose and corticosteroids were supplied. He got clear consciousness after symptomatic treatment. The patient, then, received gamma knife radiosurgery for pituitary tumor in the Second Hospital of Beijing Armed Police. The symptom of dizziness was getting better after the surgery and light sensation of the left eye recovered. Postoperative replacement therapy of hydrocortisone $20 \mathrm{mg} /$ day and euthyrox $25 \mu \mathrm{g} /$ day was treated, and no symptoms of episodes of dizziness occurred. The patient was manifested with dryness of the mouth, polydipsia symptoms on January 2011, whose water deprivation test results were indicated in Table 1.

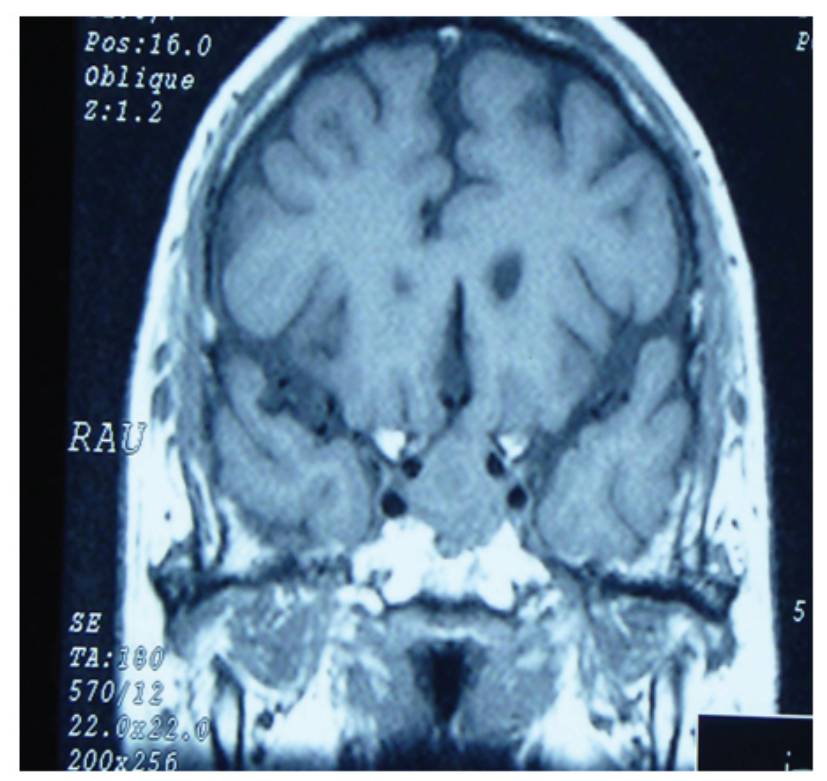

Figure 2: Reexamination results of pituitary MRI

The diagnosis of part of central diabetes insipidus was confirmed according to the experimental results. Its symptoms of dry mouth, polydipsia was relieved when Minirin 1/day was treated and intake volume of water was about 1,500 2,500 ml, urine volume was 800-1,500 ml.

\subsection{Confirmation of the diagnosis}

(1) Pituitary adenoma

(2) Anterior pituitary hypofunction

(3) Secondary adrenal hypofunction 
(4) Hypothyroidism

(6) Pituitary apoplexy

(5) The central part of the diabetes insipidus

Table 1: Water deprivation test results

\begin{tabular}{|c|c|c|c|c|c|c|c|}
\hline Time & $\begin{array}{l}\text { Urine volume } \\
\text { (ml) }\end{array}$ & $\begin{array}{l}\text { Blood osmotic } \\
\text { pressure (mOsm) }\end{array}$ & Weight (Kg) & $\begin{array}{l}\text { Blood pressure } \\
(\mathrm{mmHg})\end{array}$ & $\begin{array}{l}\text { Heart rate } \\
\text { (Times/min) }\end{array}$ & $\begin{array}{l}\text { Urine specific } \\
\text { (gravity) }\end{array}$ & $\begin{array}{l}\text { Urine osmotic } \\
\text { pressure (mOsm) }\end{array}$ \\
\hline $6 a m$ & 400 & 215 & 65.5 & $120 / 70$ & 62 & 1.005 & 215 \\
\hline $7 \mathrm{am}$ & 400 & 209 & 65 & $120 / 70$ & 62 & 1.005 & 209 \\
\hline 8am & 50 & 261 & 65 & $115 / 70$ & 63 & 1.005 & 261 \\
\hline 9am & 30 & 297 & 65.5 & $120 / 70$ & 66 & 1.005 & 297 \\
\hline 10am & 40 & 304 & 65.5 & $120 / 70$ & 56 & 1.005 & 304 \\
\hline 10:10am & $\begin{array}{l}\text { Subcutaneous of } \\
\text { Vasopressin } 4 \mathrm{u}\end{array}$ & & & & & & \\
\hline 11:10am & 20 & 420 & 65.5 & $115 / 65$ & 60 & 1.015 & 420 \\
\hline
\end{tabular}

\section{Discussion}

\subsection{Dr. Qinxue Li}

Dr. Qinxue Li is a deputy chief physician of geriatrics department at the Third Affiliated Hospital of Inner Mongolia Medical University, specializing in the diagnosis and treatment of geriatrics endocrine.

Pituitary adenomas are tumors that occur in the pituitary gland, represents $10 \%$ of all intracranial neoplasms, mostly seen in patients aged from 20 to 50 . It is a rare thing that pituitary adenomas occurs among patients over 50 years old. Elderly patients with pituitary adenomas, therefore, only takes up $4 \%$ to $5 \%$ of all the pituitary adenomas cases. ${ }^{[1]}$ The clinical study found that pituitary tumors of different ages were characterized with different symptoms. The symptoms of pituitary adenomas in elderly patients differ widely from those among younger patients from the perspective of clinical manifestations, imaging features and some of the biological behavior of tumors. A series of endocrine dysfunction, called pituitary dysfunction syndrome, is likely to appear, mainly involving glands of the gonads, the thyroid and adrenal cortex when all or most of the posterior pituitary is destroyed. When over $50 \%$ of the pituitary tissue is destroyed, clinical symptoms are stimulated. The symptoms are evident when the damage is up to $75 \%$. It may develop serious symptoms if $95 \%$ was damaged. The clinical symptoms were hidden among elderly patients with recession due to its lower metabolism and slow reaction to the environment.

The patient in the case went to the doctor due to fatigue, dizziness, nausea, vomiting, hyponatremia and lower blood pressure with complex and diverse clinical manifestations, where may lead to misdiagnosis and missed diagnosis. It is mainly because: (1) Atypical clinical manifestations. Pituitary adenoma is complex and varied, especially among elderly patients, and no specific symptoms are found in non-secreting adenomas. (2) Non-secreting adenomas may potentially lead to fatigue, weakness, listlessness, loss of appetite, lethargy, hypoglycemia, hyponatremia and other symptoms by stimulating gonadotropin, thyroid stimulating hormone and adrenocorticotropic hormone secretion, which are often misdiagnosed by non-specialist. Pituitary dysfunction is suspected when the signs of unexplained fatigue, lethargy, trichomadesis, loss or disappearance of libido appear so that it is better to have ACTH, PRL, TSH, LH, FSH and other hormones checked for early diagnosis to reduce misdiagnosis rate. (3) Hyponatremia. The abnormal changes of sodium induced by other disease are often neglected, therefore, clinical multi-faceted scientific thinking to seek the cause of hyponatremia in elderly patients is essential and the use of supplemental screening should be performed on the basis of the patients' description of the disease. The disease is associated with gastroenterology, neurology, psychiatry, cardiology, hematology, ophthalmology.

Pituitary adenomas among elderly patients are nonfunctional adenomas, found to be large volume with evident invasiveness and higher incidence of tumor stroke. The patient usually seeks medical help when the tumor larger cause intracranial hypertension and optic nerve compression symptoms as the early clinical manifestations are not typical. As a matter of fact, the existence of the tumor seems longer than the clinical courses. Anatomically pituitary tumors are classified by their size based on radiological findings; either microadenomas (less than $10 \mathrm{~mm}$ ) or macroadenomas (equal or greater than $10 \mathrm{~mm}$ ). Generally, the larger the tumor grows, the less blood supply to the tumor so that tumors stroke takes up a higher proportion and tumor volume increased rapidly in the short term, known as severe acute onset of clinical manifestation. ${ }^{[2]}$ Pituitary apoplexy, a clinical syndrome, refers to the sudden hemorrhage or infarction in pituitary adenoma initiates the outbreak increase of tumor volume, then further results in the increase presser of the saddle, and oppresses the surrounding structure or adjacent tissue. The patient in the case died of PA of pituitary adenoma. Sheehan et al. reported that the occurrence of pituitary apoplexy among elderly patients over 70 takes up $12 \%{ }^{[3]}$ Clinical manifestations of PA depends on tumor expansive direction, bleeding rate, blood loss, the degree of compression of surrounding peripheral nerve and damages 
to the pituitary. Different types own different manifestations. Shamim et al. reported that $60 \%$ of patients were with headache or visual impairment. ${ }^{[4]}$

\subsection{Dr. Dan Zhao}

Dr. Dan Zhao is an attending doctor of Geriatrics Department at the Third Affiliated Hospital of Inner Mongolia Medical University, specializing in the diagnosis and treatment of geriatrics.

Pathogenesis of pituitary tumors is a complex multi-step process with many factors get involved, and it remains to be identified. Ras oncogene belongs to proto-oncogene family, including proteins H-ras, K-ras and N-ras, and the encoded protein has GTP activity. Ras mutations generally occur in invasive pituitary adenomas. Lin et al. ${ }^{[5]}$ detected Ras mutations in $7 \%$ of invasive pituitary tumors but none in non-invasive pituitary adenomas. Ras mutations are much associated with the occurrence and invasion of pituitary adenomas. Pituitary tumor-transforming gene (PTTG) plays an important role in the formation and development of pituitary adenomas. PTTG-centered web determines the proliferation network and cell division. Abnormal expression of PTTG has a bearing on the occurrence and invasion of pituitary adenomas. ${ }^{[6]}$ PTTG is highly expressed in about $90 \%$ of pituitary adenomas, but it does not express or presents low expression in normal pituitary tissues. The occurrence and development of pituitary adenomas is a complex process of interaction with more factors involved, including activation of oncogenes and inactivation of tumor suppressor genes, and abnormal cell signaling pathway. Many methylation of tumor suppressor genes and other related gene silencing may contribute to the generation of pituitary adenoma, but the mechanism of pituitary adenoma is unclear. A further study pituitary adenoma pathogenesis, therefore, is essential for a better understanding of diagnosis, treatment and prognosis of pituitary adenoma.

\subsection{Dr. Ying Wang}

Dr. Ying Wang is an attending doctor of Geriatrics Department at the Third Affiliated Hospital of Inner Mongolia Medical University, specializing in the diagnosis and treatment of geriatrics.

Pituitary adenoma is a benign, slow-growing (mostly expensive growth) tumor, may compress surrounding structures, and few may direct invade adjacent structures. Its clinical manifestations mainly include the following two aspects:

(1) The symptom presented by adjacent tissue structures due to the increase of tumor volume: headache is the most frequent manifestation. It is largely associated with the increase of the tumor volume, because of which causing in- creased pressure within the sella, stimulating brain blood vessels, nerves, meninges and so on. As the disease progresses, the tumor grows outside the sella, which may reduce the pressure and then relieve the symptom of headache. Besides that, the patients also complained about the symptoms of vision loss and visual field defects. A variety of clinical reports indicate that the incidence of the vision loss and visual field defects is $50 \%$ to $100 \%$ among patients with non-functioning pituitary tumors. The symptom of visual field defects is usually accompanied by impaired vision, which may aggravate to blindness in severe cases. Huge tumor may extend downward thalamus so that hypothalamus dysfunction may occur, which lead to patient feeding center disorders (anorexia, bulimia), thermoregulation disorders, diabetes insipidus.

(2) Anterior pituitary induced by the compression and destruction of pituitary tissues beyond tumors. Most multiple pituitary hormone deficiency phenomenon will emerge by and by, firstly presented with the absence of LH, FSH, GH and PRL. Most male patients will have sexual hypoactivity and impotence, then develop into the gradual emergence of secondary sexual characteristics, such as pubic hair, armpit hair, beard off, sparse, testicular atrophy as the disease progresses. Thyroid-stimulating hormone deficiency comes second, such as the performance of apathy, low body temperature, dry skin, bradycardia, fatigue, hypotension accompanied by ACTH deficiency. ${ }^{[7]}$ About $10 \%$ of patients are with full-pituitary dysfunction. However, secondary adrenal insufficiency induced by ACTH deficiency would initiate obvious clinical manifestations when stress situations are encountered. The patient showed full-pituitary dysfunction, adrenal gland, thyroid impaired functions, emerged with diabetes insipidus.

\subsection{Dr. Ruijing Yan}

Dr. Ruijing Yan is a resident doctor of Geriatrics Department at the Third Affiliated Hospital of Inner Mongolia Medical University, specializing in the diagnosis and treatment of geriatrics.

The diagnosis of pituitary tumors should be distinguished from the following diseases. (1) Proliferation. Primary hypothyroidism and adrenal insufficiency lead to pituitary hyperplasia. Thyroid function tests showed TSH elevated and $\mathrm{FT}_{4}$ decreased significantly. MRI examination showed that pituitary enlarged equably, and homogeneous enhance after enhanced scan. (2) Tumors. 1) Craniopharyngioma. It may occur at all ages, mostly seen among children and teenagers. In addition to visual impairments and visual deficits, arrest of growth and development, hypoplasty of sexual organs, obesity and diabetes insipidus are more likely to occur due to pituitary reduced function and hypothalamus involvement. Tumors of larger volume usually lead to the increase of intracranial pressure. Diagnostic imaging demon- 
strated that tumors would be seen with cystic change and calcification. The main tumors were located on the saddle, and pituitary tissue in the bottom of the saddle. 2) Germcell tumor. It mostly occurs in children, with rapid progression and evident clinical symptoms, characterized by diabetes insipidus, precocious puberty, weight loss and pituitary dysfunction. Imaging showed that lesions were located in the saddle, reinforcing effect was obvious. Blood HCG and HCG of cerebrospinal fluid elevated in some patients, and they were sensitive to radiotherapy. Besides that, pituitary tumors should be distinguished from granulosacell tumor, pituitary metastatic cancer, lymphoma, hamartoma, nerve sheath tumors and teratomas. (3) Inflammation: 1) Lymphocytic hypophysitis. The disease is more commonly seen in women of childbearing age, with unknown origin, may be an autoimmune disease caused by a virus. It is mainly manifested with diabetes insipidus, partially characterized by hypopituitarism. The diagnostic imaging revealed that pituitary stalk significantly enlarged and pituitary tissue increased to varying degrees. 2) Pituitary abscess. The symptoms are recurrent fever, headache, significantly loss of vision, associated with other cranial nerve damage with rapid progression of the disease. The diagnostic imaging demonstrates that the lesion volume of the disease is not large, which is not inconsistent with the clinical symptoms. Soft tissue structures surrounding sella enhanced obviously. Pituitary adenomas and other diseases need to be differentiated, such as empty sella syndrome, suprasellar germ cell tumors, pituitary metastasis, the internal carotid artery aneurysm.

\subsection{Dr. Gaisheng Tian}

Dr. Gaisheng Tian is a director of Geriatrics Department at the Third Affiliated Hospital of Inner Mongolia Medical University, specializing in the diagnosis and treatment of geriatrics endocrine.

Pathogenesis of pituitary tumors is more complex, involving inherent defects of the pituitary tumor cells, paracrine and autocrine dysfunction, and other aspects of hypothalamic regulation disorders. The primary treatment method of the disease includes surgery, drug therapy and radiation therapy, among which surgery is more preferred, especially for non-functional adenomas. Surgical treatment can be divided into craniotomy and transsphenoidal minimally invasive surgery. The latter is widely accepted by the clinicians due to good effect and safety. Currently, craniotomy is required when significant expansion at saddle and invasion to subarachnoid occur, and transsphenoidal surgery is available for the rest cases and would achieve good results. ${ }^{[8]}$ However, elderly patients, who received complete surgical resection is less due to the larger tumor volume as well as the invasiveness to cavernous sinus. As the elderly patients usually have poor tolerance to the surgery and the tumor grow slowly so that there is no need to do the complete resection, which may bring risk inevitably. Based on the above-mentioned factors, transsphenoidal surgery is more applicable for elderly patients. Postoperative residual tumor can be treated with radiotherapy and satisfactory tumor control could be obtained. ${ }^{[9]}$ Radiotherapy imposes better effect on non-functioning adenomas, but produces poor effect on other types. ${ }^{[10]}$ Pituitary radiation therapy not only prevents further tumor growth but also cuts down the hormone levels of increased secretion. It has good effect on the courses of pituitary tumor growth control treatment. ${ }^{[11]}$

The patient in this case received radiation therapy rather than surgery due to the fact that he is older for the surgery, the tumor was large and total resection rate was lower. Good results were achieved by the treatment. Thyroid and adrenal therapy proves to be alternative treatment when fullpituitary dysfunction appears.

PA is broadly defined as the syndrome induced by hemorrhage and vascular occlusion of pituitary gland or pituitary adenoma, including sudden headache, visual disturbances, muscle paralysis, unconsciousness and lack of pituitary hormone secretion. ${ }^{[12]}$ However, most of the literature believes that it is caused by pituitary vascular occlusion or bleeding, so is called pituitary apoplexy. ${ }^{[13]}$ It is easily mistaken for other intracranial disease as the clinical manifestations vary, sometimes, coma and even death may occur in some cases due to the rapid progress of the disease. The key to the treatment of pituitary apoplexy depends on early and accurate diagnosis. PA is a rare and fatal disease. However, it carries a higher survival rate if the diagnosis of PA was taken into consideration and a rapid treatment was performed. PA, therefore, may be the potentially cause for unexplained hypoglycemia, uncorrected hyponatremia or shock, and unexplained coma. Pituitary apoplexy treatment includes medication and surgery. Acute adrenal insufficiency is prone to occur so that the application of the corticosteroids should be performed immediately, such as immediate intravenous injection of hydrocortisone $100 \mathrm{mg}$, and additional application was employed every 6-8 h. Electrolyte must be closely monitored when signs of diabetes insipidus appear.

Pituitary tumor is the most frequent disease of all the intracranial tumors, whose clinical manifestations are complex and varies so that misdiagnosis and missed diagnosis is easily to occur. There is no specific symptom at the early phase, and patients usually go to the doctor for fatigue, dizziness, nausea, vomiting, hyponatremia and low hypertension. Therefore, clinical multi-faceted scientific thinking to seek the cause of hyponatremia in elderly patients is essential and the use of supplemental screening should be performed on the basis of the patients' description of the disease. As a clinician, we should have a solid foundation, of which a master of our own professional disease, and of which a comprehension of diseases related with profession as well. 


\section{References}

[1] Hu Jia, Shengtao Lv, Xinguo Cui, et al. The clinical and pathological analysis of the pituitary adenoma in the elderly. Chinese journal of gerontology. 2010; 4(30): 538-539.

[2] Hiroshi Nishioka, Jo Haraoka, Kiyohiro Akada, et al. Growth potential ofprolactinomas in men: is it really different from women. Surg Neu-rol. 2003; 59(5): 386-391. http://dx.doi.org/10.1016/ S0090-3019(03) 00012-0

[3] Sheehan JM, Douds GL, Hill K, et al. Transsphenoidalsurgery for pituitary adenoma in elderly patients. Acta Neurochir(Wien). 2008; 150(6): 571-574. PMid:18414774. http://dx.doi.org/ 10.1007/s00701-008-1581-2

[4] Shamim MS, Bari ME, Khursheed F, et al. Pituitaryadenomas:Presentations and outcomes in a South Asiancountry. Can J Neurol Sci. 2008; 35(2): 198-203. PMid:18574934. http://dx. doi.org/10.1017/S0317167100008635

[5] Lin Y, Jiang XF, Shen Y, et al. Frequentmutations and amplifications of the PIK3CA gene in pituitary tumors. EndocrRelat Cancer. 2009; 16(1): 301-310. PMid:18852163. http://dx.doi.org/ 10.1677/ERC-08-0167

[6] Filippella M, Galland F, Kujas M, et al. Pituitary tumourtransforming gene (PTTG) expression correlates with the proliferative activity and recurrence status ofpituitary adeno-mas: a clin- ical and mi munohistochemical study. Clin Endocrinol (Oxf). 2006; 65(4): 536-543. PMid:16984249. http://dx.doi.org/ $10.1111 / \mathrm{j} .1365-2265.2006 .02630 . \mathrm{x}$

[7] Jiawei Chen. Adenohypophysis hypofunction. Rengao Ren, Zaiying Lu. Internal medicine. Version 6. Beijing: People's medical publishing house; 2004. 698-699 p.

[8] AtulGoe 1, Dattatraya Muzumdar. Surgical management of giant pituitary adenomas. Inter Congress Series. 2004; 1259: 99-106.

[9] Sun HP, Beverly D, Gerg B. Integration of surgery with fractionated stereotactic radiotherapy for treatment of non-functioning pituitary macroadenomas. Radiation Oncology Bio. 2005; 3(61): 795-808.

[10] Kobayashi T. Long term results of stereotactic gamma knife Radiosurgery for pituitary adenomas. Specific strategies for different types of adenoma. Prog Neurol Surg. 2009; 22: 77-95. PMid:18948721.

[11] Jiang Feng, Jianmin Zhu, Ziqiang Jiang. Pituitary adenomas gamma knife treatment of follow-up study. Jiangxi medical. 2007; 42(1): 20-21.

[12] Schwedt TJ, Matharu MS, Dodick DW. Thunderclap headache. Lancet Neurol. 2006; 5(7): 621-631. http://dx.doi.org/10. 1016/S1474-4422(06) 70497-5

[13] Chuang CC, Chang CN, Wei KC, et al. Surgical treatment for severe visual compromised patients after pituitary apoplexy. J Neurooncol. 2006; 80(1): 39-47. PMid:16645717. http://dx.doi.org/10. $1007 / \mathrm{s} 11060-006-9148-7$ 